\title{
THE ELECTRIC DIPOLE MOMENT OF W-BOSON
}

\author{
Darwin CHANG ${ }^{1.2}$, Wai-Yee KEUNG ${ }^{2.3}$ and Jiang LIU ${ }^{4}$ \\ 'Department of Physics and Astronomy, Northwestern Unicersity, Ecanston, IL 60201, USA \\ ${ }^{2}$ Fermilab, P.O. Box 500, Batavia, IL 60510, USA \\ ${ }^{3}$ Department of Physics, Unicersity of Illinois at Chicago, IL 60680, USA \\ ${ }^{4}$ Randall Laboratory of Physics, Unicersity of Michigan, Ann Arbor, MI 48109, USA
}

Received 20 July 1990

\begin{abstract}
Theoretical predictions of the electric dipole moment $D_{\mathrm{w}}$ of the $\mathrm{W}$ gauge boson are estimated for various models of $\boldsymbol{C P}$-violation. It is shown that, for the supersymmetric model and the Weinberg-Higgs model, $D_{\mathrm{w}}$ can be of the order of $10^{-20} \mathrm{ecm}$, which is close to the upper bound derived indirectly from the neutron electric dipole moment. We also obtain smaller $D_{\mathrm{w}}$-values of about $10^{-22} e \mathrm{~cm}$ and less than $10^{-38} e \mathrm{~cm}$, predicted by the left-right model and the Kobayashi-Maskawa (KM) model, respectively.
\end{abstract}

\section{Introduction}

In the past twenty six years since the discovery of $C P$ violation [1], even with a lot of efforts, little progress has been made in determining where this violation arises in the elementary particle interactions [2]. Immediately after the discovery of $C P$-violation, it was suggested by Salzman and Salzman [3] and others [4] that the observed $C P$-violation in the neutral kaon system might result from an intrinsic electric dipole moment of $\mathrm{W}$, denoted by $D_{\mathrm{w}}$ hereafter. Indeed, the order of magnitude of the $C P$-violation parameter $\epsilon$ in the kaon decay is very close to $\alpha / \pi$ and hence this suggested a $C P$-violating electromagnetic effect on the weak interaction amplitudes. It was also realized that a finite $D_{\mathrm{w}}$ could induce an electric dipole moment for the neutron. More recently, Marciano and Queijeiro [5] updated the original analysis of Salzman and Salzman and they found that measurements of the neutron electric dipole moment of the order $10^{-25} \mathrm{e} \mathrm{cm}$ could be used to place a very stringent upper bound on $D_{\mathrm{w}}$ for its absolute magnitude,

$$
D_{\mathrm{w}} \leqq 10^{-20} e \mathrm{~cm} .
$$

They have assumed a reasonable form factor to tame the divergence. Unless this form factor suppression is much stronger, the restriction eliminates the possibility of using $D_{\mathrm{w}}$ to explain $\epsilon$. However, the effect of $D_{\mathrm{w}}$ of the order of $10^{-20} \mathrm{ecm}$ 
may still be accessible [6] in certain processes, for instance in the scattering $\gamma \mathrm{e}^{ \pm} \rightarrow \mathrm{W}^{ \pm} \boldsymbol{\nu}$, for future experiments. Also, careful studies of the polar and azimuthal distributions of leptons and antileptons produced in $W$ decays [7] may further provide useful constraints on the size of $D_{\mathrm{w}}$. With the increasing production luminosity of $\mathrm{W}$ pairs in laboratories, it becomes of current interest to estimate the size of $D_{\mathrm{w}}$ in various $C P$-violating gauge models.

\section{A general expression for $D_{w}$}

It is well known that if $\boldsymbol{P}$ - and $\boldsymbol{T}$-symmetries are violated, elementary particles with spin degrees of freedom may have electric dipole moments. The most general form of the W-boson coupled to a photon has seven terms [7], among them two of which violate $P$ - and $T$ - and hence $C P$-symmetries,

$$
i k W_{\mu}^{\dagger} W_{v} \tilde{F}^{\mu v}+i\left(\lambda / M_{\mathrm{W}}^{2}\right) W_{\alpha \mu}^{\dagger} W_{l}^{\mu} \tilde{F}^{v \alpha} .
$$

Here $W_{\mu}$ is the $\mathrm{W}^{-}$gauge potential, $W_{\mu v}=\partial_{\mu} W_{v}-\partial_{v} W_{\mu}+\ldots$, and the dual of the photon field strength is $\bar{F}^{\mu v}=\frac{1}{2} \epsilon^{\mu v \alpha \beta}\left(\partial_{\mu} A_{v}-\partial_{v} A_{\mu}\right)$. In the momentum space, these terms can be expressed as

$$
f_{1} \epsilon^{\mu v \alpha \beta}\left(p-p^{\prime}\right)_{\beta}+\left(f_{2} / M_{\mathrm{W}}^{2}\right) \epsilon^{\mu \cdot \rho \beta}\left(p-p^{\prime}\right)_{\beta}\left(p+p^{\prime}\right)^{\alpha}\left(p+p^{\prime}\right)_{\rho} .
$$

Here $p$ and $p^{\prime}$ are the incoming and the outgoing momenta of the W-boson. The form factors $f_{1}=\lambda-k$ and $f_{2}=\frac{1}{2} \lambda$ are functions of $\left(p-p^{\prime}\right)^{2}$ (the square of the momentum transfer). The electric dipole moment $D_{\mathrm{w}}$ can be expressed [7] in terms of these form factors in the limit $\left(p-p^{\prime}\right)^{2} \rightarrow 0$ in the unit of $e / 2 M_{\mathrm{w}}=1.2$ $\times 10^{-16} e \mathrm{~cm}$,

$$
D_{\mathrm{w}}=\left(f_{1}-4 f_{2}\right)\left(e / 2 M_{\mathrm{w}}\right) .
$$

In gauge theories, a $C P$-violating but $S U(2)_{L}$-invariant term $\theta W^{\mu \nu} \tilde{W}_{\mu \nu}$ can be added to the lagrangian. However, this term can be rewritten as a total divergence and thus will not contribute to $D_{\mathrm{w}}$ perturbatively. Nonperturbative effects due to such a term are suppressed at least by a factor [8] $\exp \left(-8 \pi^{2} / g^{2}\right)$, where $g$ is the weak coupling constant, and hence extremely small. In what follows we will ignore this contribution. Also, we realize that the first term in eq. (2) has a dimensionality of four. However, this term is not invariant under $S U(2)_{L} \times U(1)$ and, therefore, can only be generated through higher-dimensional gauge-invariant terms of the form $\phi^{n} W^{\dagger} W \tilde{A}$. Here $\phi^{n}$ represents, generically, an interaction of $n$ neutral Higgs fields. Since the other term already has a dimensionality of six, the $C P$-violating electromagnetic form factor is thus induced by operators with a dimensionality greater than four. As a result, $D_{\mathrm{w}}$ is calculable even in models with "hard" $C P$-violation. 


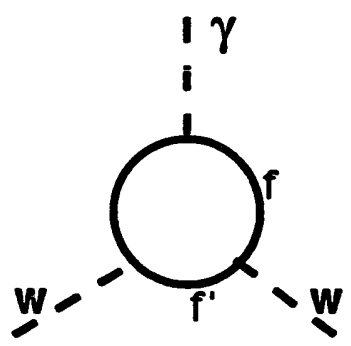

Fig. 1. One-loop Feynman graphs for calculating $D_{\mathrm{w}}$ due to the left-handed and the right-handed currents.

An obvious distinction between the $\boldsymbol{C P}$-conserving and the $\boldsymbol{C P}$-violating electromagnetic form factors of $\mathrm{W}$ is that the $\boldsymbol{C P}$-violating terms are directly proportional to the Levi-Civita tensor. Such a tensor occurs naturally in the spinor trace of the Dirac matrices, $\epsilon^{\mu \nu \alpha \beta}=\frac{1}{4} i \operatorname{Tr}\left(\gamma^{\mu} \gamma^{\nu} \gamma^{\alpha} \gamma^{\beta} \gamma_{5}\right)$. Thus the fermion loop is required to give $D_{\mathrm{w}}$. This fact can be understood in a different way. A perturbative renormalizable theory that contains only gauge bosons and Higgs bosons (without fermions) is always invariant under the symmetry $\tilde{P}: x^{\mu} \rightarrow x_{\mu}, W^{\mu} \rightarrow W_{\mu}, \phi \rightarrow \phi$. Consequently, the lowest-order contribution that may potentially contribute to $D_{\mathrm{w}}$ must contain fermion loops. To study the size of $D_{w}$ quantitatively, we consider the foilowing general $\mathrm{W}$-fermion interaction:

$$
\mathscr{L}^{\mathrm{cc}}=-\frac{g}{\sqrt{2}} W_{\mu}^{+} \sum_{i, j} \bar{f}_{i} \gamma^{\mu}\left(V_{i j} L+U_{i j} R\right) f_{j}^{\prime}
$$

Here $L, R=\frac{1}{2}\left(1 \mp \gamma_{5}\right), i$ and $j$ are generation indices, $f$ and $f^{\prime}$ represent fermion fields with charges different by one unit. The phases in the mixing matrices $V$ and $U$ are the sources of the $C P$-violation. For simplicity we will not consider the lepto-quark theories where the mixing between quark and lepton is not zero. The one-loop contributions to $D_{\mathrm{w}}$ are depicted in fig. 1. Evaluating these graphs, we find

$D_{\mathrm{w}}=\frac{g^{2} C}{8 \pi^{2}} \sum_{a, i, j} \frac{e}{2 M_{\mathrm{w}}} \frac{m_{i} m_{j}^{\prime}}{M_{\mathrm{w}}^{2}} \operatorname{Im}\left(V_{i j} U_{i j}^{*}\right)\left(Q_{i} \mathscr{I}\left(\frac{m_{i}^{2}}{M_{\mathrm{w}}^{2}}, \frac{m_{j}^{\prime 2}}{M_{\mathrm{w}}^{2}}\right)+Q_{j}^{\prime} \mathscr{I}\left(\frac{m_{j}^{\prime 2}}{M_{\mathrm{w}}^{2}}, \frac{m_{i}^{2}}{M_{\mathrm{w}}^{2}}\right)\right)$,

where

$$
\mathscr{S}(x, y)=\int_{0}^{1} \mathrm{~d} \alpha \frac{\alpha}{\alpha(\alpha-1)+\alpha x+(1-\alpha) y-i \epsilon}
$$


Here $Q_{i}$ and $Q_{j}^{\prime}$ are the charges for fermions $f$ and $f^{\prime}$, respectively. The color factor $C$ is 1 or 3 for the lepton or for the quark. The fermion masses $m_{i}$ and $m_{j}^{\prime}$ occur explicitly in the factor $m_{i} m_{j}^{\prime} / M_{\mathrm{W}}^{2}$ due to the helicity argument. The above expression can be integrated directly. When $\Delta \equiv 1+x^{2}+y^{2}-2 x-2 y-2 x y \leqslant 0$,

$$
\mathscr{I}(x, y)=\frac{1}{2} \ln \frac{x}{y}+\frac{1-x+y}{\sqrt{-\Delta}}\left(\arctan \frac{1+x-y}{\sqrt{-\Delta}}+\arctan \frac{1+y-x}{\sqrt{-\Delta}}\right) .
$$

A typical value of this function at the electroweak scale is about unity, e.g. $\mathscr{F}(1,1)=\sqrt{3} \pi / 9 \simeq 0.6$. When $\Delta>0$,

$\mathscr{I}(x, y)=\Delta^{-1 / 2}\left[z_{+} \ln \left|z_{+}^{-1}-1\right|-z_{-} \ln \left|z_{-}^{-1}-1\right|-i \pi(1-x+y) \theta(1-\sqrt{x}-\sqrt{y})\right]$,

where $z_{ \pm}=\frac{1}{2}(1-x+y \pm \sqrt{\Delta})$. As indicated by the step function $\theta$, for sufficiently small values of $x$ and $y$ (i.e. $1>\sqrt{x}+\sqrt{y}$ ), the internal fermions can be or-shell and as a result $\mathscr{I}(x, y)$ picks up an imaginary part. However, in contrast to the $C P$-violating phases, this "loop" phase will not change its sign under the hermitian conjugation. In the absence of $C P$-violation, $\operatorname{Im}\left(V_{i j} U_{i j}^{*}\right)=0$, we have $D_{\mathrm{w}}=0$ as it should.

\section{The values of $D_{w}$ in some theories of $C P$-violation}

(1) In the KM model [9], the electric dipole moment vanishes at one-loop level because there is no right-handed current $\left(U_{i j}=0\right)$. The reason is very similar to that of calculating the neutron electric dipole moment [10]: finite value of $D_{\mathrm{w}}$ in the $\mathrm{KM}$ model can only appear at least from two-loop diagrams. Including the GIM cancellation, we estimate that

$$
D_{\mathrm{w}}(\mathrm{KM} \text { model }) \leq s_{1} s_{2} s_{3} s_{\delta}\left(\frac{g^{2}}{8 \pi^{2}}\right)^{2}\left(\frac{e}{2 M_{\mathrm{W}}}\right) \frac{m_{\mathrm{b}}^{4} m_{\mathrm{s}}^{2} m_{\mathrm{c}}^{2}}{M_{\mathrm{W}}^{8}} \leq 10^{-38} e \mathrm{~cm} .
$$

Here $s_{1} s_{2} s_{3} s_{\delta}$ is a combination of $C P$-violating factors of the order $10^{-4}$. The t-quark mass $m_{\mathrm{t}}$ at the scale of $M_{\mathrm{W}}$ has been assumed. In fact, one can argue that even at the two-loop level the contribution is probably zero. The argument goes as follows. There are only four fermion lines in the two-loop diagram. In the unitary gauge, all the interactions are left handed, therefore the quark masses must appear quadratically. In the $\mathrm{KM}$ model, the $C P$-violation disappears when any two of the up- or the down-type quarks are degenerate in mass. Therefore we expect any $C P$-violating effect to carry a factor of $\Pi_{i<j}\left(m_{\mathrm{d}_{i}}^{2}-m_{\mathrm{d}_{j}}^{2}\right)\left(m_{\mathrm{u}_{i}}^{2}-m_{\mathrm{u}_{j}}^{2}\right)$. As a result, there are a total of 6 powers of quadratic mass differences. The GIM effect at each 

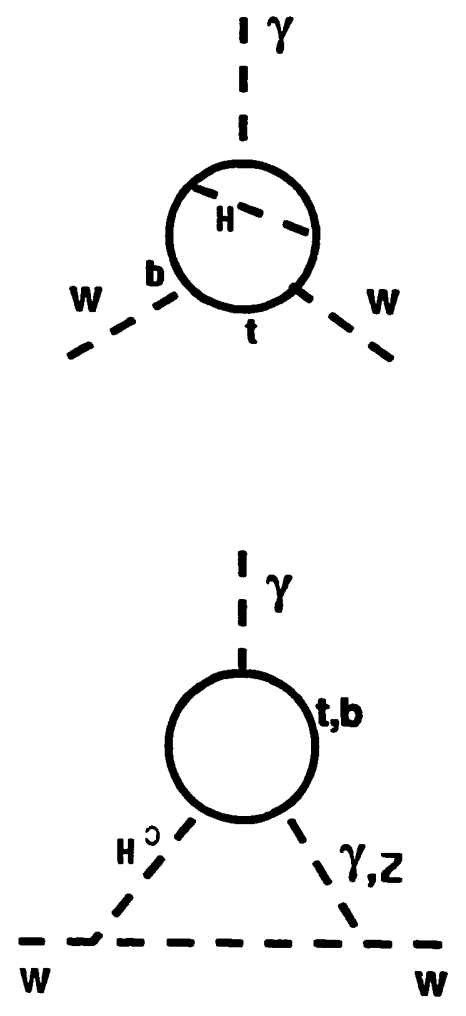

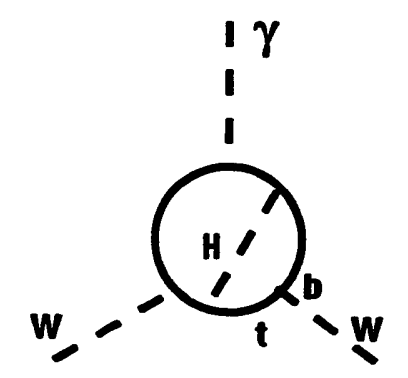

(a)

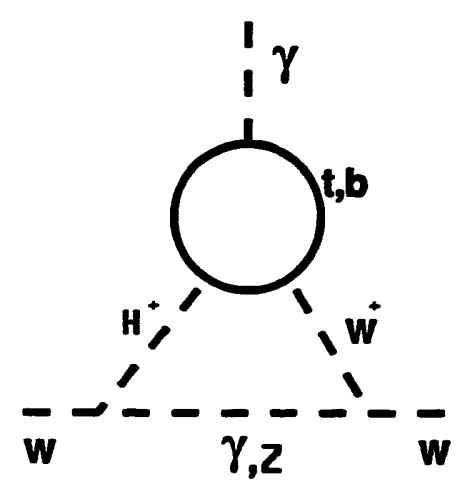

(b)

Fig. 2. A sample of two-loop Feynman graphs for calculating $D_{w}$ in the Weinberg-Higgs model.

fermion line in the loop yields one factor of quadratic mass differences. Therefore, from a two-loop diagram, it can only produce four powers of quadratic mass differences which is smaller than the $\mathbf{6}$ powers as needed for $\boldsymbol{C P}$-violation.

(2) In the Weinberg-Higgs model [11], $\operatorname{Im}\left(V_{i j}\right)=0$ and $U_{i j}=0$. Consequently, $D_{\mathrm{w}}=0$ at one-loop level. However, a nonzero $D_{\mathrm{w}}$ can arise through two-loop graphs. The dominant graphs are shown in fig. 2 where the exchanged Higgs boson can be charged or neutral. Some of the neutral Higgs contributions (fig. 2a) have recently been studied by $\mathrm{He}$ and McKellar [12]. The complete two-loop amplitude still requires more detailed calculations. Here we only estimate the size of $D_{w}$ to be of the order

$$
D_{\mathrm{W}} \sim \sin \delta_{\mathrm{H}}\left(\frac{g^{2}}{8 \pi^{2}}\right)^{2}\left(\frac{m_{\mathrm{f}}}{M_{\mathrm{H}^{0}}}\right)^{2}\left(\frac{e}{2 M_{\mathrm{W}}}\right) .
$$

The $C P$-violating phase $\delta_{\mathrm{H}}$ characterizes the complex mixing in the Higgs sector. It could be of the order of unity. The contribution is large if the mass $m_{\mathrm{H}^{\prime \prime}}$ of the 
neutral Higgs is small. With three generations of fermions, it gives

$$
D_{\mathrm{W}}(\text { Higgs model }) \leq 10^{-20}\left(\frac{m_{\mathrm{t}}}{100 \mathrm{GeV}}\right)^{2}\left(\frac{10 \mathrm{GeV}}{m_{\mathrm{H}}}\right)^{2} e \mathrm{~cm} .
$$

For $m_{\mathrm{t}}>100 \mathrm{GeV}$ and $m_{\mathrm{H}^{0}} \sim 10 \mathrm{GeV}$, this yields $D_{\mathrm{W}} \leqq 10^{-20} e \mathrm{~cm}$.

(3) In left-right models [13], the leading contribution should be due to the $C P$-violating phase associated with the left-right mixing. The electric dipole moment $D_{\mathrm{w}}$ arises even for the case of only one generation. We can consider the dominant contribution from the top and the bottom quark generation (assuming $g_{\mathrm{L}}=g_{\mathrm{R}}$ ).

$$
\operatorname{Im}\left(V_{\mathrm{tb}} U_{\mathrm{tb}}^{*}\right) \simeq \xi \sin \delta_{\mathrm{LR}},
$$

where $\xi$ is the left--right mixing which is bound [14] by $\xi \leq 5 \times 10^{-3}$. We find the dominant contribution is of the order

$$
\begin{aligned}
D_{\mathrm{W}}(\text { LR model })= & \xi \sin \delta_{\mathrm{LR}} \frac{g^{2}}{8 \pi^{2}} \frac{m_{\mathrm{b}} m_{\mathrm{t}}}{M_{\mathrm{W}}^{2}}\left(\frac{e}{2 M_{\mathrm{W}}}\right) \\
& \times\left[2 \mathscr{I}\left(\frac{m_{\mathrm{t}}^{2}}{M_{\mathrm{W}}^{2}}, \frac{m_{\mathrm{b}}^{2}}{M_{\mathrm{W}}^{2}}\right)-\mathscr{I}\left(\frac{m_{\mathrm{b}}^{2}}{M_{\mathrm{W}}^{2}}, \frac{m_{\mathrm{t}}^{2}}{M_{\mathrm{W}}^{2}}\right)\right] \leqslant 10^{-22} e \mathrm{~cm} .
\end{aligned}
$$

The numerical analysis for the $t-b$ quark contribution is shown in fig. 3. One should be aware of potential off-diagonal contribution. Again, a much larger

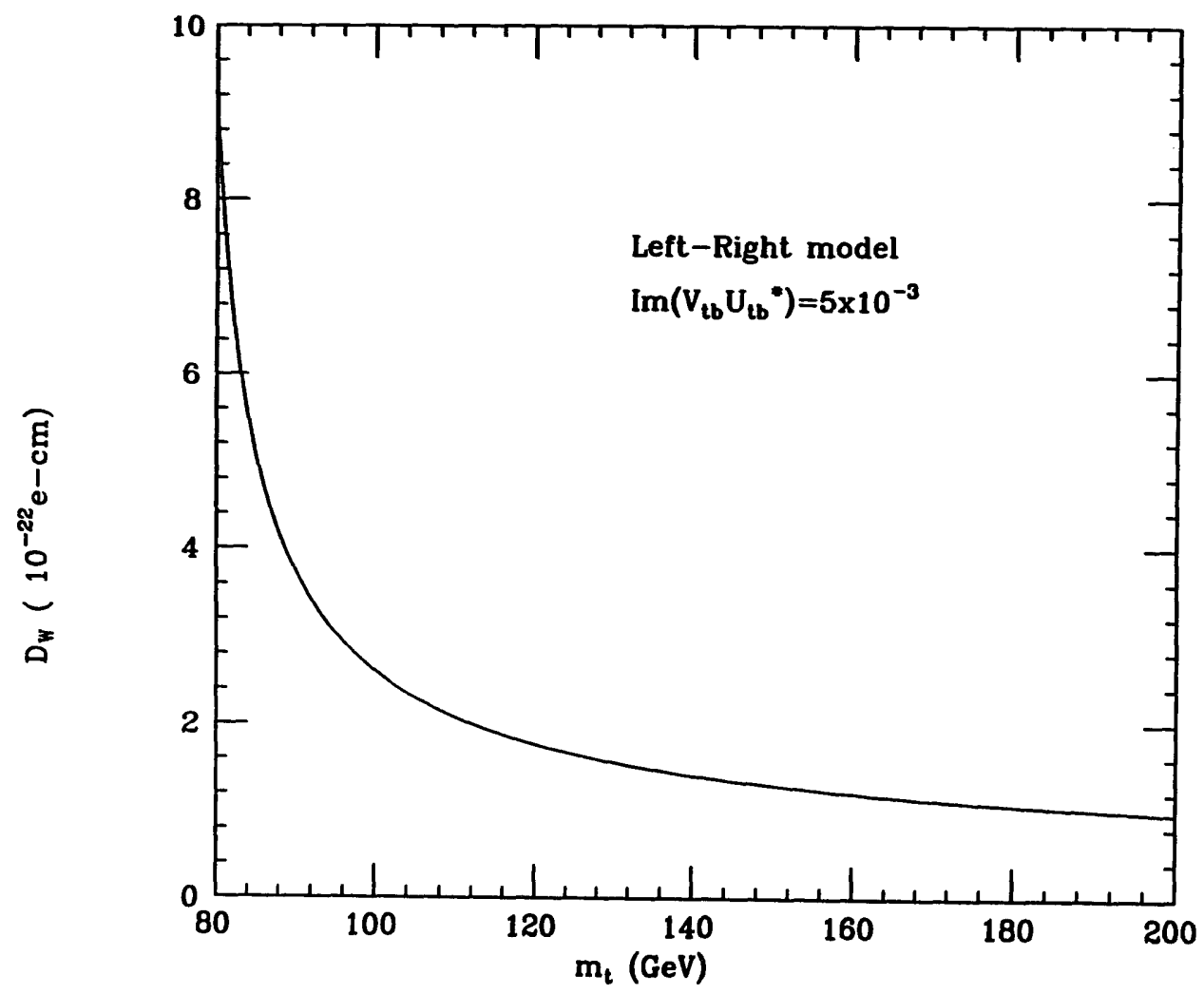

Fig. 3. $D_{\mathrm{W}}$ in the left-right model due to the t-b generation, assuming $\xi \sin \delta_{\mathrm{LR}}=5 \times 10^{-3}$. 
contribution could arise (like in the Weinberg-Higgs model discussed above) through Higgs boson exchanges if the specific model has a complicated Higgs structure.

(4) In supersymmetric (SUSY) models [15], the internal fermions in fig. 1 can be supersymmetric particles - the charginos and the neutralinos. To show the basic mechanism of the $C P$-violation, we consider only the case when the neutralino is the photino $\bar{\gamma}$ and the chargino is the wino $\omega$. In general, the photino will mix with the neutral higgsino, the zino and the neutrinos; and the wino will mix with the charged higgsino. We avoid this extra complication in the simplified scenario in order to illustrate the physics involved. One can easily extend our approach to the general case. In terms of the independent Weyl's fields $\omega_{L}^{+}, \omega_{L}^{-}$and $\tilde{\gamma}_{L}$, the relevant lagrangian is

$$
\mathscr{L}=-e W_{\mu}^{+}\left(\overline{\omega_{L}^{+}} \gamma^{\mu} \tilde{\gamma}_{L}+\overline{\tilde{\gamma}_{L}} \gamma^{\mu} \omega_{L}^{-}\right)-m_{\bar{\gamma}} \overline{\bar{\gamma}_{L}^{c}} \bar{\gamma}_{L}-m_{\omega} \mathrm{e}^{i \delta_{j}} \overline{\omega_{L}^{+}} \omega_{L}^{-c}+\ldots+\text { h.c. }
$$

The mass terms of the wino and the photino break supersymmetry softly. A phase $\delta_{\mathrm{S}}$ in the mass term is usually allowed and it cannot be totally absorbed by redefining the fields. Consequently, this causes $C P$ non-conservation. To recover the usual form of the mass expression, we define the Dirac field $\omega^{+}=\omega_{L}^{+}+$ $\mathrm{e}^{i \delta_{S}}\left(\omega_{\mathrm{L}}^{-}\right)^{\mathrm{c}}$ and the Majorana field $\tilde{\gamma}=\tilde{\gamma}_{\mathrm{L}}+\tilde{\gamma}_{\mathrm{L}}^{\mathrm{c}}$. The above lagrangian becomes

$$
\mathscr{L}=-e W_{\mu}^{+} \overline{\omega^{+}+\gamma^{\mu}}\left(L-\mathrm{e}^{i \delta_{\mathrm{S}}} R\right) \tilde{\gamma}-\frac{1}{2} m_{\bar{\gamma}} \overline{\tilde{\gamma}} \tilde{\gamma}-m_{\omega^{\omega^{+}} \omega^{+}+\ldots}
$$

Both the left-handed and the right-handed currents appear with a relative phase $\delta_{\mathrm{s}}$. We can obtain $D_{\mathrm{w}}$ from eqs. (5)-(7),

$$
D_{\mathrm{W}}=\frac{e^{2}}{4 \pi^{2}}\left(\frac{e}{2 M_{\mathrm{w}}}\right) \frac{m_{\tilde{\gamma}} m_{\omega}}{M_{\mathrm{W}}^{2}} \sin \delta_{\mathrm{s}} \mathscr{I}\left(\frac{m_{\omega}^{2}}{M_{\mathrm{w}}^{2}}, \frac{m_{\tilde{\gamma}}^{2}}{M_{\mathrm{W}}^{2}}\right) .
$$

Usually there could be additional factors due to the mixings among charginos and neutralinos. At present, no direct phenomenological constraint on these mixings is available. Also, the $C P$-violating phase $\delta_{s}$, allowed by the soft supersymmetry breaking lagrangian, could be naturally of the order of unity. The only natural suppression on $D_{\mathrm{W}}$ in this class of models is thus the loop factor $e^{2} / 4 \pi^{2}$. As a result, we expect that in supersymmetric models $D_{\mathrm{w}}$ could be as large as the present limit given by eq. (1),

$$
D_{\mathrm{W}}(\text { SUSY model }) \leq 10^{-20} e \mathrm{~cm} .
$$

(5) In mirror models (for a recent review see ref. [16]), the presence of mirror quarks and mirror leptons introduces right-handed currents with $\mathrm{W}$. The mixing between a quark and its mirror image, $\xi_{q}$, is strongly constrained by the absence of 
flavor changing neutral current, where one finds [17]

$$
\xi_{\mathrm{q}} \lesssim 10^{-3}-10^{-4} \text {. }
$$

The relevant lagrangian can be written as

$$
\begin{aligned}
\mathscr{L}= & -\frac{g}{\sqrt{2}} W_{\mu}\left(\overline{u_{\mathrm{L}}} \gamma^{\mu} d_{\mathrm{L}}+\overline{U_{\mathrm{R}}} \gamma^{\mu} D_{\mathrm{R}}\right) \\
& +M_{\mathrm{u}} \overline{u_{\mathrm{R}}} U_{\mathrm{L}}+M_{\mathrm{d}} \overline{d_{\mathrm{R}}} D_{\mathrm{L}}+M_{2}\left(\overline{u_{\mathrm{L}}} U_{\mathrm{R}}+\overline{d_{\mathrm{L}}} D_{\mathrm{R}}\right) \\
& +m_{\mathrm{u}} \overline{u_{\mathrm{L}}} u_{\mathrm{R}}+m_{\mathrm{d}} \overline{d_{\mathrm{L}}} d_{\mathrm{R}}+m_{\mathrm{U}} \overline{U_{\mathrm{L}}} U_{\mathrm{R}}+m_{\mathrm{D}} \overline{D_{\mathrm{L}}} D_{\mathrm{R}}+\ldots
\end{aligned}
$$

Note that $M_{i}$ are $\mathrm{SU}(2)_{\mathrm{L}}$-invariant masses and $m_{i}$ are $\mathrm{SU}(2)_{\mathrm{L}}$ broken masses. It is reasonable to assume that $m_{\mathrm{u}}, \boldsymbol{m}_{\mathrm{d}}$ are the smallest massive parameters. Also, the condition $m_{\mathrm{U}}, m_{\mathrm{D}} \gg M_{\mathrm{u}, \mathrm{d}, 2}$ is imposed for the constraint of mirror mixing (eq. (19)). It is possible to define the fields so that only $m_{u}, m_{d}$ are complex, i.e. $\boldsymbol{C P}$-violating parameters. Therefore the $\boldsymbol{C P}$-violating effect should be proportional to either $m_{\mathrm{u}}$ or $m_{\mathrm{d}}$. Now, it is easy to draw diagrams that will contribute to $D_{\mathrm{w}}$. Some typical ones are shown in fig. 4. Each $M_{i}$ insertion corresponds a factor of $\xi_{\mathrm{q}}$ mixing. It then follows from eqs. (5)-(7) that contributions to $D_{\mathrm{w}}$ from virtural quark-mirror-quark exchange is typically of the order

$$
D_{\mathrm{W}}(\text { mirror model }) \sim \frac{g^{2}}{8 \pi^{2}} \xi_{\mathrm{q}}^{2} \sin \delta_{\mathrm{M}}\left(\frac{e}{2 M_{\mathrm{W}}}\right) \frac{m_{\mathrm{q}}}{m_{\mathrm{Q}}} \leq 10^{-25} e \mathrm{~cm}
$$

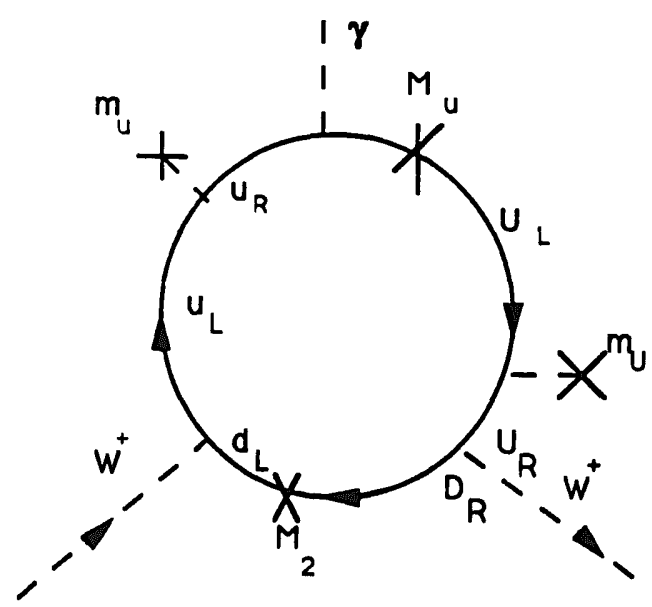

Fig. 4. Typical graph for $D_{\mathrm{W}}$ in the mirror models with the relevant mass insertions illustrated. Here $C P$-violation requires all parameters in the $\mathrm{u}-\mathrm{U}($ or $\mathrm{d}-\mathrm{D}$ ) mass matrix appear. 
TABLE 1

Upper limit of $D_{\mathrm{w}}$ in various $C P$-violation models. Here it is assumed that (1) there are only three generation of fermions and (2) in left-right and mirror models Higgs contributions to $D_{W}$ are negligible

\begin{tabular}{cc}
\hline$C P$-violation models & Upper limit on $D_{\mathrm{w}}(e \mathrm{~cm})$ \\
\hline KM & $10^{-38}$ \\
Weinberg-Higgs & $10^{-20}$ \\
left-right & $10^{-22}$ \\
SUSY & $10^{-20}$ \\
mirror & $10^{-25}$ \\
\hline
\end{tabular}

The phase $\delta_{M}$ characterizes the complex mixing among the quark and its mirror. A similar size of contribution can also be generated from lepton-mirror-lepton mixings. It should also be pointed out that the constraint on $\xi_{\mathrm{q}}$ can be evaded if (1) there is a fourth generation and (2) its mixing with the rest of the generations are negligible. In that case, we find $D_{\mathrm{w}}$ can be as large as of the order $10^{-20} e \mathrm{~cm}$.

\section{Conclusion}

Our results for $D_{\mathrm{w}}$ in different $C P$-violation models are summarized in table 1 . Here it is assumed that there are only three generations of fermions and the Higgs contribution to $D_{\mathrm{w}}$ is negligible except in models where $C P$-violation is due to the Higgs boson exchange. In SUSY and Higgs models, we find that $D_{w}$ could be as large as the present upper limit while in other models it appears to be small.

This research was supported in part by the US Department of Energy. While we were finalizing this work, we received a preprint by Atwood et al. [18], who studied $D_{\mathrm{w}}$ in the left-right model.

\section{References}

[1] J. Christenson, J. Cronin, V. Fitch and R. Turlay, Phys. Rev. Lett. D13 (1964) 138

[2] L. Wolfenstein, Annu. Rev. Nucl. Part. Sci. 36 (1986) 137

[3] F. Salzman and G. Salzman, Phys. Lett. 15 (1965) 91; Nuovo Cimento 41A (1966) 443

[4] J. Bernstein, G. Feinberg and T.D. Lee, Phys. Rev. 139 (1965) 1650;

S. Barshay, Phys. Lett. 17 (1965) 78;

B.A. Arbuzov and A.T. Fillipov, Phys. Lett. 20 (1966) 537

[5] W.J. Marciar:o and A. Queijeiro, Phys. Rev. D33 (1986) 3449

[6] A. Queijeiro, Phys. Lett. B193 (1987) 354

[7] K. Hagiwara, P.D. Peccei and X. Zeppenfeld, Nucl. Phys. B282 (1987) 253

[8] T.P. Cheng and L.-F. Li, Gauge theory of elementary particle physics (Oxford Univ. Press, Oxford, 1984).

[9] M. Kobayashi and T. Maskawa, Prog. Theor. Phys. 49 (1973) 652

[10] E.P. Shabalin, Sov. Phys. Usp. 26 (1983) 4 
[11] S. Weinberg, Phys. Rev. Lett. 37 (1976) 657

[12] X.-G. He and B. McKellar, Melbourne preprint UM-P-90/39 (1990)

[13] J.C. Pati and A. Salam, Phys. Rev. D10 (1974) 275;

R.N. Mohapatra and J.C. Pati, Phys. Rev. D11 (1975) 2558;

G. Senjanović and R.N. Mohapatra, Phys. Rev. D12 (1975) 1502;

H. Fritzsch and P. Minkowski, Nucl. Phys. B103 (1976) 61

[14] I.I. Bigi and J.-M. Frère, Phys. Lett. B110 (1982) 255;

J. Donoghue and B. Holstein, Phys. Lett. B113 (1982) 382;

L. Wolfenstein, Phys. Rev. D29 (1984) 2130;

E. Masso. Phys. Rev. Lett. 52 (1984) 1956

[15] N.P. Nilles, Phys. Rep. 110 (1984) 1;

H.E. Haber and G.L. Kane, Phys. Rep. 117 (1985) 76

[16] J. Maalampi and M. Roos, Phys. Rep. 186 (1990) 53

[17] I. Umemura and K. Yamamoto, Phys. Lett. B108 (1982) 37;

K. Enqvist, K. Mursula and M. Roos, Nucl. Phys. B226 (1983) 121

[18] D. Atwood, C.P. Burgess, C. Hamzaoui, B. Irwin and J.A. Robinson, Brookhaven preprint 90-0358 (1990) 